\title{
Possibilities of holographic techniques in laser scanning microscopy
}

Yu. Zakharov, M. Muravyeva, V. Dudenkova, I. Mukhina, I. Meglinski

Yu. Zakharov, M. Muravyeva, V. Dudenkova, I. Mukhina, I. Meglinski, "Possibilities of holographic techniques in laser scanning microscopy," Proc. SPIE 9540, Novel Biophotonics Techniques and Applications III, 95400P (17 July 2015); doi: 10.1117/12.2183814

SPIE. Event: European Conferences on Biomedical Optics, 2015, Munich, Germany 


\title{
Possibilities of holographic techniques in laser scanning microscopy
}

\author{
Yu.Zakharov *a, b , M.Muravyeva ${ }^{\text {a }}$, V. Dudenkova ${ }^{\text {a }}$, I.Mukhina ${ }^{\text {a,c }}$, I.Meglinski ${ }^{\text {d }}$ \\ ${ }^{a}$ Lobachevsky State University of Nizhni Novgorod, Nizhny Novgorod, Russia \\ ${ }^{\mathrm{b}}$ Center for Advanced Biomedical Imaging and Photonics, Harvard University, Boston, MA, USA \\ ${ }^{\mathrm{c}}$ Nizhny Novgorod State Medical Academy, Nizhny Novgorod, Russia \\ ${ }^{\mathrm{d}}$ Opto-Electronics and Measurement Techniques Laboratory, University of Oulu, Oulu, Finland
}

\begin{abstract}
Holographic scanning microscopy - novel technique both in laser scanning microscopy and digital holographic microscopy allow multimodal approach to cell and tissue investigation in biomedical applications promising new advantages (quantitative phase imaging, superresolution, computerized tomography), but regular reconstruction leads to incorrectness. Analysis of light propagation through the schematics allows to offer reconstruction procedures depending on recording conditions.
\end{abstract}

Keywords: Digital holography, scanning microscopy, computerized optical tomography

\section{INTRODUCTION}

Laser scanning microscopy obtains an image by means of point-by-point acquest, digitizing and inlaying a mosaic picture [1]. At that image features such as transversal dimensions or intensity distribution can be measured, and image quality depends on abilities of optics, scanning system and detector.

For both image analysis and image processing attention must be paid to reliability and accuracy of measured results. Mainly they are determinated by the point spread function (PSF). Its longitudinal size depends on the length of the focal volume and scattering properties of the object. Laser scanning confocal microscopy withdraws the second source of PSF extension, and in the case of fluorescence confocal microscopy multiphoton excitation reduce optical slice depth even greater. Moreover some techniques, using scanning of image plane by very small steps and processing by appropriate software, allows to overcome diffraction limit in transverse direction.

As for longitudinal size measurements different interferometric techniques ensure it on the level of a few nanometers, one of them is holographic interferometry in its digital construction [2]. But now all of them have realized only for widefield microscopy [e.g. 3, 4], whereas scanning confocal regime give some grate opportunities in functional imaging of living cells. A novel approach both to digital holography and laser scanning microscopy (LSM) - holographic scanning microscopy (HSM) [5] combine usual LSM techniques (e.g. fluorescence and/or confocal microscopy) with quantitative phase microscopy by recording digital hologram during pointwise scanning of the sample.

\section{DESIGN OF HOLOGRAPHIC SCANNING MICROSCOPE}

In order to enrich capabilities of laser scanning microscope by digital hologram recording it is necessary to add reference beam pathway to microscope design. In the case of our Zeiss LSM-510 microscope beam passing through main beam splitter MDBS (marked out on the Figure 1 by bold dash line) had not used before. We have included to the microscope construction reference beam mirror RBM. Now this beam reflects from the RBM, comes back and it is reflected by MDBS toward PMT. In such a way it forms reference wave. In our case we should place reference beam mirror outside scan module in order to equalize signal and reference beams path length.

Novel Biophotonics Techniques and Applications III, edited by Arjen Amelink, I. Alex Vitkin,

Proc. of SPIE-OSA Biomedical Optics, SPIE Vol. 9540, 95400P · (c) 2015 SPIE-OSA

CCC code: $1605-7422 / 15 / \$ 18 \cdot$ doi: $10.1117 / 12.2183814$ 


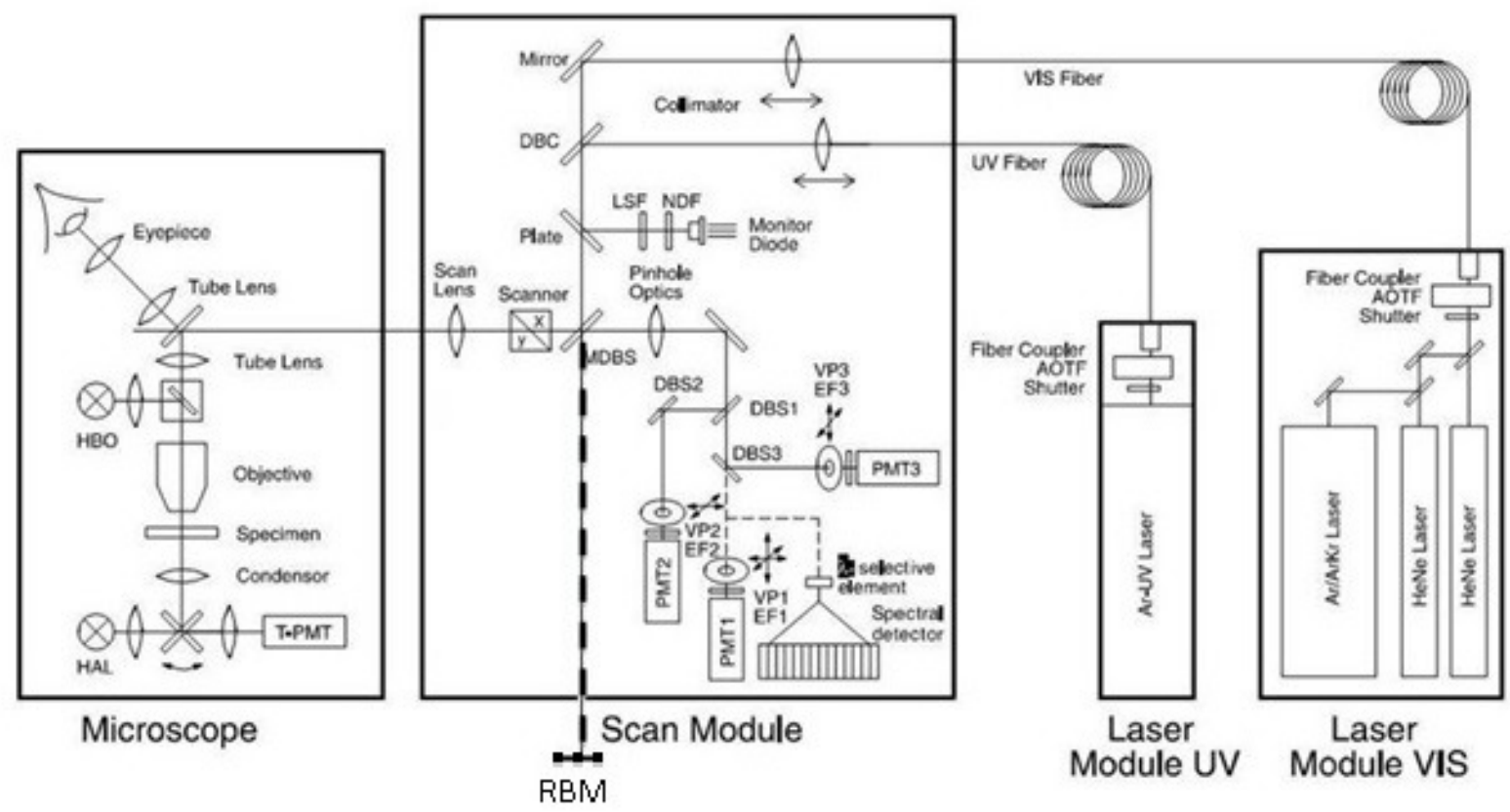

Figure 1. Schematic of holographic scanning microscope on the base of Zeiss LSM-510. Bold dash line show beam using as reference. RBM is reference beam mirror added to regular microscope design, which transform usual LSM to HSM.

Reference wave interfere with reflected from specimen object wave and resulting intensity of light is transformed by PMT and analog-digital converter to one pixel of the discrete hologram at each scanning step. Its amount depends on not only intensity of object and reference waves but their phase difference too. In this way whole scan stack create digital hologram. We name this technique as holographic scanning microscopy (HSM). It may be said that it is focused-image hologram because of image plane and hologram plane are optically conjugated, but features of object illumination and light propagation in confocal microscopy configuration leads to difference in hologram structure for holographic scanning microscopy and usual wide-field focused-image digital holography including wide-field holographic microscopy (WFHM). Each point of scanning area is illuminated under each own direction, so object wave from each point of sample has different subsidiary phase incursion. So HSM-hologram can't be reconstructed by usual algorithm and necessitate special reconstructing processing.

Moreover integral phase incursion of wave front in HSM differs from integral phase incursion for transparent object in WFHM due to the fact that only light reflected (scattered) in objective lens focal volume gives significant contribution to detector lighting. This feature gives opportunity to obtain tomographic data without angle scanning but by Z-scan series as well as to separate contribution of refractive index distribution and path length inside sample. However reconstruction algorithm should be specific again.

Additional advantage is simple combining of confocal reflectance and fluorescence microscopy with quantitative phase microscopy by recording of fluorescent signals and hologram in the different channels of modernized LSM scan module.

\section{DESCRIPTION OF HOLOGRAM RECORDING AND RECONSTRUCTION IN HSM}

\section{Coherent light propagation through confocal scanning microscope}

To analyze features of digital hologram forming by designed holographic microscope we consider schematics presented on Figure 2 including all specific parts. Since holography is principal coherent process we use radiooptic approach for image formation in coherent wave fields [6].Complex amplitude transformation of propagating wave is calculated by convolution with paraxial approximation of pulse response of free space: $h(z)=\sqrt{\frac{1}{i \lambda z}} \exp \left(\frac{\pi}{\lambda} \cdot i \cdot \frac{x^{2}}{z}\right)$, where $\lambda$ is wave length,,$z$ is the length of free space piece, $i$ is imaginary unit, $x$ is transversal coordinate. 
Objective and confocal lenses was considered as theoretical. Because of scan module changes the direction of illumination for every point of scanning area signal wave has phase additions that are different for every image pixel. They are determinated by scanning mirror inclination.

In result, signal wave for $k, l$ scanning position is described by expression

$$
S_{k l}(x, z)=-\frac{2 \pi}{\sqrt{2} \cdot \sqrt{\lambda F_{2}}} \cdot \delta\left(-\frac{2 \pi}{\lambda F_{2}} x\right) \cdot\left(R_{k l} \cdot \exp \left(i\left(2 \varphi_{k l}+\left(\frac{2 \pi}{\lambda} F_{1}-\frac{2 \pi}{\lambda} z_{2}-\frac{4 \pi}{\lambda} z_{3}\right) \cdot \sin ^{2} \theta_{k l}+\frac{\pi}{\lambda \cdot F_{2}} \cdot x^{2}\right)\right),\right.
$$

where $F_{1}$ is objective lens focal distance; $F_{2}$ is confocal lens focal distance; $z_{2}$ is the distance from scanning mirror to objective lense; $z_{3}$ is the distance from scanner to confocal lens; $\theta_{k l}$ is scanning mirror incline angle; $\varphi_{k l}$ is phase distortions caused by scan module; $\delta(\ldots)$ is point spread function; $R_{k l}=r_{k l} \exp \left(i \psi_{k l}\right)$ is specimen complex reflectance.

Reference wave is described by expression

$$
A(x, z)=-\frac{2 \pi}{\sqrt{2 \cdot \lambda F_{2}}} \cdot \delta\left(-\frac{2 \pi}{\lambda \cdot F_{2}} \cdot x\right) \cdot \exp \left(i \frac{\pi}{\lambda \cdot F_{2}} \cdot x^{2}\right) .
$$

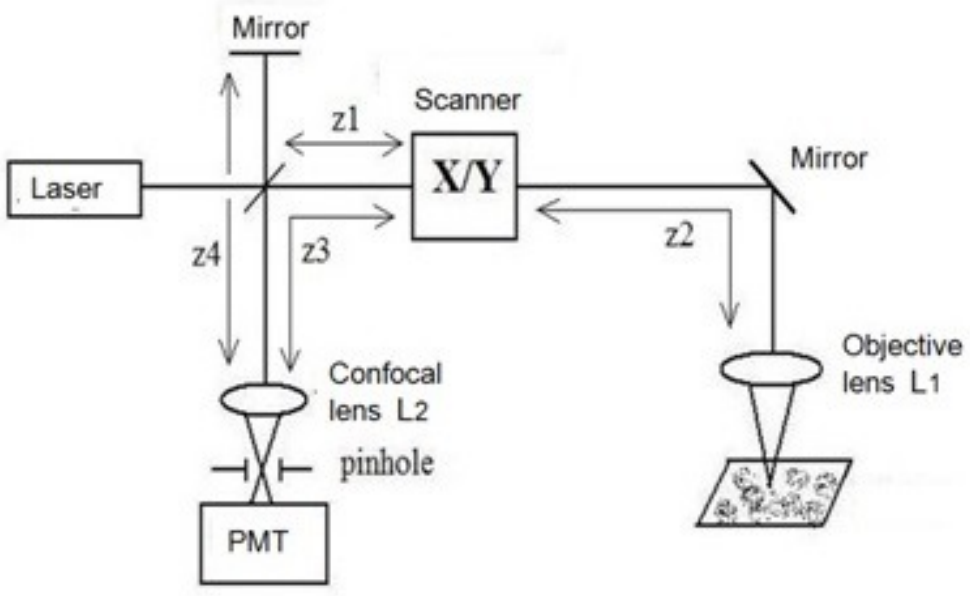

Figure 2. Schematics of holographic channel of the multimodal LSM-HSM microscope.

\section{Hologram creation and wave front reconstruction}

Hologram pixel is formed as integral energy in pinhole during the exposure time of sum of the signal and reference:

$$
H_{k l}=\iint_{S_{\text {pin }} t_{\text {exp }}}\left|S_{k l}+A\right|^{2} d x d t
$$

that is described by expression

$$
H_{k l}=A^{2}+Q^{2} r_{k l}^{2}+Q A \cdot r_{k l} \cdot\left[\exp \left\{i\left(\psi_{k l}+2 \varphi_{k l}+P \sin ^{2} \theta_{k l}\right)\right\}+\exp \left\{-i\left(\psi_{k l}+2 \varphi_{k l}+P \sin ^{2} \theta_{k l}\right)\right\}\right.
$$

and can be represented as

$$
H_{k l}=A^{2}+Q^{2} r_{k l}^{2}+Q R_{k l} \cdot A \exp \left\{i \gamma_{k l}\right\}+Q \widehat{R}_{k l} \cdot A \exp \left\{-i \gamma_{k l}\right\}
$$

where $\hat{R}_{k l}$ is complex conjugate to $R_{k l}, \gamma_{\mathrm{kl}}=2 \varphi_{\mathrm{kl}}+\mathrm{P}_{\sin ^{2}} \theta_{\mathrm{kl}}, \mathrm{P}=\frac{2 \pi}{\lambda} \mathrm{F}_{1}-\frac{2 \pi}{\lambda} \mathrm{z}_{2}-\frac{4 \pi}{\lambda} \mathrm{z}_{3}, Q$ is real constant.

Whole scan stack create digital hologram. (5) shows that compare to regular wide-field hologram structure interference terms have phase distortions. So such digital hologram reconstructed by regular algorithm (for example double Fouriertransforms with filtering in the space frequencies domain) gives incorrect phase portrait of object. 
Considering pixel numbers $k, l$ as coordinates in hologram plane, let us perform Fourier transform of hologram structure:

$$
\begin{gathered}
\mathscr{F}\{H(k, I)\}=\mathscr{F}\left\{A^{2}+Q^{2} r^{2}(k, I)\right\}+\mathscr{F}\{Q R(k, I) \cdot A \exp \{i \gamma(k, I)\}\}+\mathscr{F}\{Q \hat{R}(k, I) \cdot A \exp \{-i \gamma(k, I)\}\}= \\
=\mathscr{F}\left\{A^{2}+Q^{2} r^{2}(k, I)\right\}+A Q \cdot \mathcal{F}\{R(k, I)\}^{*} \mathscr{F}\{A \exp \{i \gamma(k, I)\}\}+\mathscr{F}\{\hat{R}(k, I)\} * \mathcal{F}\{A \exp \{-i \gamma(k, I)\}\}
\end{gathered}
$$

where symbol $*$ means convolution.

If three items of (6) are separated in space frequency domain, the first and the third of them can be filtered out, but then instead of usual for such algorithm spectrum centering and invert Fourier transform, before invert Fourier transform it is necessary to perform deconvolution with the $\mathscr{F}\{A \exp \{i \gamma(k, I)\}\}$ core.

For this it is needed to find this function or at least every $\gamma_{\mathrm{kl}}=2 \varphi_{\mathrm{kl}}+\mathrm{P}_{\sin ^{2}} \theta_{\mathrm{kl}}$ to combine it.

It is possible with the help of hologram recording of mirror by the same setup:

$$
H_{M}(k, I)=Q^{2}+A^{2}+2 Q A \cdot \cos \left(2 \varphi(k, I)+P \sin ^{2} \theta(k, I)\right),
$$

and intensity acquisition of such signal $\left(\mathrm{Q}^{2}\right)$ and reference $\left(A^{2}\right)$ beams.

In result $\cos ^{-1}\left\{\frac{(7)-\left(\mathrm{Q}^{2}+\mathrm{A}^{2}\right)}{2 \mathrm{QA}}\right\}$ gives us $\gamma(k, l)$.

Thus, we can correctly reconstruct scanned hologram for accurate phase measurements with the help of HSM. If items in (6) are not absolutely separated in space frequency domain it is necessary to use another specified reconstruction process.

\section{Acknowledgments}

The study is supported by RFBR grants 13-02-1420 and 15-42-02658.

\section{REFERENCES}

[1] Webb R.H., "Confocal optical microscopy”, Rep. Prog. Phys. 59, 427-471 (1996).

[2] Kim M.K., "Principles and techniques of digital holographic microscopy". SPIE Reviews. 1, 018005-1 018005-50 (2010).

[3] Zakharov Yu., Rybnikov A., Dudenkova V., Muravyeva M., Mitroshina E., Mukhina I., "Investigation of neuronal culture cell alteration with the help of digital holographic interferometer attached to laser scanning microscope." Optics in the Life Science (Bio-Optics:Design and Application). Technical Digest. JT2A.22. OSA (2013).

[4] Zakharov Yu. "Micro-holography upgrade of fluorescence microscope for multi-modal investigation of living neuron cells and circuits", OSA Technical Digest . Biomedical Optics (BIOMED) 2014 paper: BS4B.4.

[5] Zakharov Yu., Muravyeva M., Dudenkova V., Mukhina I., Vitkin E., and Perelman L., "Holographic scanning microscopy - novel approach to digital holography and laser scanning microscopy," in Imaging and Applied Optics 2014, OSA Technical Digest, paper DW5B.1, (2014).

[6] Zverev V.A., [Physical Foundation of Image Formation by Wave Fields], Nizhny Novgorod, IAP RAN, 252 p. (1998). 